Pacific Journal of Mathematics

THE ZERO DIVISOR CONJECTURE FOR SOME SOLVABLE 


\section{THE ZERO DIVISOR CONJECTURE FOR SOME SOLVABLE GROUPS}

\section{ROBERT L. SNIDER}

Let $F$ be a field and $G$ a group. The zero divisor conjecture states that if $G$ is torsion free, then the group algebra $F[G]$ is torsion free. A series of papers by various authors have resulted in a proof of this conjecture for polycyclic-by-finite groups. The next most natural step would seem to be groups which are poly-(torsion free rank one abelian)-by-finite. These are pcecisely the solvable groups of finite cohomological dimension. A perhaps more attractive description of these groups is the solvable-by-finite subgroups of $G L_{n}(Q), Q$ being the rational numbers. We are able to prove this conjecture for the class of these groups where the primes in the finite "top" are different from the primes that make the rank one abelian factors non finitely generated. The key ingredient in the proof is a localization theorem which makes these non-Noetherian group rings Noetherian.

If $A$ is a finite rank torson free abelian group, pick a free abelian subgroup $F$ such that $A / F$ is torsion. The spectrum (spec $A$ ) is the set of primes $p$ such that the $p$-primary component of $A / F$ is infinite [14, p. 167]. If $N$ is a torsion free finite rank nilpotent group, $\operatorname{Spec}(N)$ is defined to be the union of the spectrum of the factors of the lower central series. We prove the

THEOREM A. Let $G$ be a torsion free group with normal subgroups $N$ and $H$ such that $N$ is finite rank nilpotent, $H / N$ is abelian, and $[G: H]<\infty$. If char $F=0$ and $\pi(G / H) \cap \operatorname{Spec}(N)=\varnothing$, then $F[G]$ has no zero divisors.

Theorem $\mathrm{A}$ is a generalization of the known result for polycyclic-by-finite groups [4]. The theorem for polycyclic-by-finite groups is known in all characteristics [3].

If one removes the condition that $\pi(G / H) \cap \operatorname{Spec} N=\varnothing$, then the groups of Theorem A are precisely the solvable-by-finite subgroups of $G L_{n}(Q), Q$ the rationals $[8,9,11]$. Also these are the solvable-by-finite groups of finite cohomological dimension [7, p. 155].

The main tool needed to prove this result is a localization theorem which then allows one to apply the techniques of [4] to prove Theorem A.

Let $N$ be a torsion free nilpotent group. Let $S=F[N]-\omega(N)$ where $\omega(N)$ is the augmentation ideal of $F[N]$. If $N$ is finitely 
generated, one can form the localization $S^{-1} F[N][13$, p. 496]. Since the general $N$ is a union of its finitely generated subgroups, it is easy to see that $S^{-1} F[N]$ always exists.

THeOREM B. If $N$ is a finite rank torsion free nilpotent group and $\operatorname{char} F=0$ or char $F=p$ and $p \notin \operatorname{Spec}(N)$, then $S^{-1} F[N]$ is Noetherian.

Theorem B is a theorem of Brewer, Costa, and Lady when $N$ is abelian [1].

In $\S 1$, we deduce Theorem A from Theorem B. Theorem B is then proved in $\S 2$.

This paper is essentially a continuation of [4]. The reader should be familiar with that paper.

1. Zero divisors. We first need an easy lemma that was pointed out to me by K. A. Brown.

Lemma 1. Suppose $H \triangleleft G$ and $[G: H]<\infty$. If $F[H]$ is an Ore domain, then $F[G]$ has no zero divisors if and only if $F[P]$ has no zero divisors for each $p$-Sylow subgroup $P / H$ of $G / H$.

Proof. Let $T$ be the nonzero elements of $F[H] . \quad T$ is an Ore set for $F[H]$ and, since $H \triangleleft G$, also for $F[G]\left[13\right.$, p. 609]. $\quad T^{-1} F[G]$ is finite dimensional over $D=T^{-1} F[H]$. Its dimension over $D$ is $[G: H]=p_{1}^{n_{1}} \cdots p_{r}^{n_{r}}$. Let $I$ be a right ideal of $T^{-1} F[G]$. If $P / H$ is a $p_{i}$-Sylow subgroup of $G / H$, then $T^{-1} F[P]$ is a division ring of dimension $p_{i}^{n_{i}}$ over $D . \quad \operatorname{dim}_{D}(I)=\operatorname{dim}_{T^{-1} F[P]}(I) \cdot \operatorname{dim}_{D} T^{-1}[P]$ whence $p_{i}^{n_{i}} \mid \operatorname{dim}_{D}(I)$. This implies $I=T^{-1} F[G]$ and $T^{-1} F[G]$ is a division ring.

The following is a slight generalization of Theorem 1 of [4]. By rank, we mean the reduced rank of a module [6].

Lemma 2. Let $R$ be a Noetherian domain and $A$ a prime ring containing $R$ (same 1). Suppose $A$ is a finite dimensional free module over $R$ with a basis $x_{1}, \cdots, x_{n}$ such that $R x_{i}=x_{i} R$. If the global dimension of $A$ is finite, then $A$ is a domain if and only if for every finitely generated projective $A$-module $P, n \mid \operatorname{rank}_{R}(P)$.

Proof. Suppose $A$ is a domain. Let $T$ be the nonzero elements of $R$. Since $R$ is Noetherian, $T$ is an Ore set. Since $R x_{i}=x_{i} R, T$ is also an Ore set for $A$. Since $A$ is a domain, $T^{-1} A$ is a division ring of dimension $n$ over $T^{-1} R$. 


$$
\begin{aligned}
\operatorname{rank}_{R}(P) & =\operatorname{rank}_{T^{-1} R}\left(T^{-1} P\right)=\operatorname{dim}_{T^{-1} R}\left(T^{-1} P\right) \\
& =\left(\operatorname{dim}_{T^{-1} R} T^{-1} A\right)\left(\operatorname{dim}_{T^{-1}} T^{-1} P\right) .
\end{aligned}
$$

Conversely, if $T$ is not a domain, there must exist a left ideal $I$ with $1 \leqq \operatorname{rank}_{R}(I)<\operatorname{rank}_{R}(T)=n$. We resolve $I$ with finitely generated projectives:

$$
0 \longrightarrow P_{n} \longrightarrow \cdots \longrightarrow P_{1} \longrightarrow P_{0} \longrightarrow I=0 \text {. }
$$

Since the reduced rank is additive on short exact sequences [6], we have $\operatorname{rank}_{R}(I)=\sum_{i=0}^{n}(-1)^{i} \operatorname{rank}_{R}\left(P_{i}\right)$. Therefore $n$ does not divide $\operatorname{rank}_{R}\left(P_{i}\right)$ for some $i$.

LEMmA 3. If $1=G_{0} \subseteq G_{1} \subseteq \cdots \subseteq G_{n}=G$ is a normal series for $G$ with torsion free abelian factors, then $F[G]$ is an Ore domain.

Proof. By induction on the length of the series, we assume that $F\left[G_{n-1}\right]$ is an Ore domain. $F[G]=\lim F[H]$ for $H / G_{n-1}$ finitely generated abelian. $F[H]$ is clearly $\overrightarrow{O r e}$ since it is an iterated twisted Laurent series ring over $F\left[G_{n-1}\right]$. Therefore $F[G]$ is an Ore domain.

Proof of Theorem A. We may assume $G$ is finitely generated. By Lemma 3, $F[H]$ is an Ore domain. By Lemma 1, we may assume $G / H$ is a $p$-group. As has been pointed out by Brown [2], it suffices to prove the theorem for fields $F$ with char $F=p$. Let $S=$ $F[N]-\omega(n)$. By Theorem B, $S^{-1} F[N]$ is Noetherian since $p \notin \operatorname{Spec}(N)$. By the proof of Lemma 13.3.5 of [13], $S$ is an Ore set for $F[H]$ and $F[G] . \quad H / N$ is finitely generated twisted Laurent series ring over $S^{-1} F[N]$ and is therefore Noetherian. Let $1=x_{1}, x_{2}, \cdots, x_{n}$ be a transversal for $H$ in $G . \quad x_{1}, \cdots, x_{n}$ is a free basis for $F[G]$ over $F[H]$ and hence for $S^{-1} F[G]$ over $S^{-1} F[H]$. Hence $S^{-1} F[G]$ is Noetherian. Also $S^{-1} F[H] x_{i}=x_{i} S^{-1} F[H]$. $G$ has finite cohomological dimension [7, p. 155]; whence $F[G]$ and also $S^{-1} F[G]$ have finite global dimension. Let $P$ be a finitely generated projective $S^{-1} F[G]-$ module. Let $F$ be a $S^{-1} F[H]$-module with trivial action. $P \otimes_{S^{-1} F[H]} F$ is an $F[G / H]$-module under an obvious action. In fact, it is a free $F[G / H]$-module since $F[G / H]$ is a local ring. Thus $[G: H]$ $\operatorname{dim}_{F}\left(P \otimes_{S^{-1} F[H]} F\right)$. By Lemma 2, the theorem will follow from the

Claim. If $P$ is a finitely generated projective $S^{-1} F[H]$-module, then $\operatorname{rank}_{S^{-1} F[H]}(P)=\operatorname{dim}_{F}\left(P \otimes_{S^{-1} F[H]} F\right)$.

To establish the claim, first note that $S^{-1} F[N]$ is a local ring and hence all projective $S^{-1} F[N]$-modules are free. Since $H / N$ is a 
finitely generated free abelian group, $S^{-1} F[H]$ is an iterated twisted Laurent series ring over $S^{-1} F[N]$. The twisted Grothendieck theorem [5] implies that all finitely generated projective $S^{-1} F[H]$ modules are stably free. Therefore $P \oplus S^{-1} F[H]^{m} \cong S^{-1} F[H]^{t}$ for some $m$ and $t$. Since rank is additive, $\operatorname{rank}(P)=t-m$. Also

$$
\begin{gathered}
\left(P \oplus S^{-1} F[H]^{m}\right) \bigotimes_{S^{-1} F^{[}[H]} F=\left[P \bigotimes_{S^{-1} F^{[}[H]} F\right] \oplus\left[S^{-1} F[H]^{m}\right] \bigotimes_{S^{-1} F[H]} F \\
\cong\left[P \bigotimes_{S^{-1} F[H]} F\right] \oplus F^{m} \cong S^{-1} F[H]^{t} \bigotimes_{S^{1} F[H]} F \cong F^{t} .
\end{gathered}
$$

Therefore $\operatorname{dim}_{F}\left[P \otimes_{S^{-1} F[H]} F\right]=t-m$ and the claim is established.

2. Localization. It is well known that if $R$ is a commutative Noetherian ring and $I$ is an ideal, then the $I$-adic completion $\hat{R}$ (i.e., the inverse $\left.\operatorname{limit} \lim R / I^{n}\right)$ is again Noetherian. It has been observed by many mathematicians [e.g., [10]] that the usual commutative proof gives the following noncommutative proposition.

Proposition 1. If $I$ is an ideal of a ring $R$ such that $R / I$ is left Noetherian and $I$ is generated by a finite number of elements of the center of $R$, then $\hat{R}$ is left Noetherian.

Lemma 4. If $R$ is a left Noetherian local ring and $I$ is an ideal generated by central elements, then every left ideal of $R$ is closed in the I-adic topology.

Proof. Since $I$ is centrally generated, it satisfies the strong Artin-Rees property by Lemma 11.2 .1 of [13]. The usual commutative proof then gives the result.

LEMMA 5. Let $G$ be a subgroup of the torsion free nilpotent group $N$. If $S=F[N]-\omega(N)$ and $T=F[G]-\omega[G]$, then $S^{-1} F[N]$ is a faithfully flat extension of $T^{-1} F[G]$.

Proof. Since $N$ is nilpotent, $G$ is a subnormal subgroup of $N$. Since a faithfully flat extension of a faithfully flat extension is faithfully flat, it suffices to consider the case when $G$ is a normal subgroup of $N$. Since $G$ is normal, the proof of Lemma 13.3 .5 of [13] implies $T$ is an Ore set for $F[N]$. Since $F[N]$ is free and hence flat over $F[G], T^{-1} F[N]$ is free and hence flat over $T^{-1} F[G]$. $S^{-1} F[G]$ is a localization of $T^{-1} F(N)$ and is therefore flat over $T^{-1} F[N]$ and hence over $T^{-1} F[G]$. The faithfulness follows from the fact that $T^{-1} F[G] / T^{-1} \omega(G)$ inbeds in $S^{-1} F[N] / S^{-1} \omega[N]$.

Proof of Theorem B. We induct on the class of the nilpotent 
group $N$. When $N$ is commutative, the result is a special case of a theorem of Brewer, Costa and Lady [1].

In general, let $Z$ be the center of $N$ and $A$ a finitely generated subgroup of $Z$ such that $Z / A$ is torsion. If char $F=p>0$, we may assume that $Z / A$ has no elements of order $p$. Let $z \in Z$. Pick $n$ minimal with $z^{n} \in A$. Then $1-z^{n}=(1-z)\left(1+z+\cdots+z^{n-1}\right) . \quad 1+$ $z+\cdots z^{n-1} \notin \omega(N)$ since $n \neq 0(\bmod p)$. Therefore $1-z \in S^{-1} F[N] \omega(A)$ whence $\quad S^{-1} F[N] \omega(A)=S^{-1} F[N] \omega(Z) . \quad S^{-1} F[N] / S^{-1} F[N] \omega(A) \cong$ $S^{-1} F[N] / S^{-1} F[N] \omega(Z) \cong S^{-1} F[N / Z]$ which is Noetherian by induction. Also since $A$ is central and finitely generated, $S^{-1} F[N] \omega(A)$ is generated by finitely many central elements. By the proposition, the $S^{-1} F[N] \omega(A)$-adic completion $\widehat{S^{-1} F[N]}$ is left Noetherian. Let $I$ be a finitely generated left ideal of $F[N]$. Let $r / 1 \in \bigcap_{n=1}^{\infty}\left(S^{-1} I+\right.$ $\left.\left(S^{-1} F[N] \omega(A)\right)^{n}\right)$. We may choose a finitely generated subgroup $G$ such that $A \subseteq G$, the generators of $I$ are contained in $F[G]$, and $r \in F[G]$. Let $T=S \cap F[G]=F[G]-\omega(G) . S^{-1} I=S^{-1} F[N](I \cap F[G])$. $F[G]$ and hence $T^{-1} F[G]$ is Noetherian. Since $S^{-1} F[N]$ is faithfully flat by Lemma 5 ,

$$
\begin{aligned}
r / 1 \in & \left.\bigcap_{n=1}^{\infty} S^{-1} F[N]\left[\left(I \cap T^{-1} F[G]\right)+T^{-1} F[G]\right) \omega(A)^{n}\right] \cap T^{-1} F[G] \\
& \left.\left.=\bigcap_{n=1}^{\infty}\left[\left(I \cap T^{-1} F\right] G\right]\right)+T^{-1} F[G] \omega(A)^{n}\right] \\
& =I \cap T^{-1} F[G] \text { by Lemma } 4 \\
& \cong S^{-1} F[N]\left(I \cap T^{-1} F[G]\right) \subseteq S^{-1} I .
\end{aligned}
$$

Therefore $I$ is closed in the $S^{-1} F[N] \omega(A)$-adic topology. With $I=0$, we have $\bigcap_{n=1}^{\infty} S^{-1} F[N] \omega(A)^{n}=0$. A noncommutative version of Theorem 31.8 of [12] now gives the theorem.

\section{REFERENCES}

1. J. W. Brewer, D. L. Coasta and E. L. Lady, Prime ideals and localization in commutative sroup rings, J. Algebra, 34 (1975), 300-308.

2. K. A. Brown, On zero divisors in group rings, Bull. London Math. Soc., 8 (976), 251-256.

3. G. H. Cliff, Zero divisors and idempotents in group rings, J. Algebra.

4. D. R. Farkas and R. L. Snider, $K_{0}$ and Noetherian group rings, J. Algebra, 42 (1976), 192-198.

5. F.T. Farrell and W. C. Hsiang, A formula for $K_{1} R_{\alpha}[T]$, in "Applications of Cate. gorial Algebra," 192-198, Proceedings of the Symposium on Pure Mathematics, Vol. 17, Amer. Math. Soc., Providence, R. I., 1970.

6. A. W. Goldie, The structure of Noetherian rings, in "Lectures on Rings and modules," 214-321, Lecture Notes in Mathematics, No. 246, Springer-Verlag, New York/ Berlin.

7. K.W. Gruenberg, Cohomological topics in group theory, Lecture Notes in Mathematics, No. 143, Springer-Verlag, New York/Berlin. 
8. V. M. Kopytov, Matrix groups, Algebra and Logic, 7 (1968), 162-166.

9. E. M. Levic, Representation of soluble groups by matrices over a field of character. istic zero, Soviet Math. Dokl., 10 (1969), 1146-1148.

10. J. C. McConnell, On completions of non-commutative Noetherian rings, Comm. in Algebra, 6 (1978), 1485-1488.

11. Ju. I. Merzljakov, On matrix representations of automorphisms, extensions, and soluble groups, Algebra and Logic, 7 (1968), 169-192.

12. M. Nagata, Local Rings, Interscience, New York, 1962.

13. D. S. Passman, The Algebraic Structure of Group Rings, Wiley-Interscience, New York, 1977.

14. D. J.S. Robinson, Finiteness conditions and generalized soluble groups II, Ergebnisse No. 62, Springer-Verlag, 1972.

Received February 20, 1979. This research was partially supported by NSF grant MCS76-05991.

VPI AND SU

Blacksburg, VA 24061 


\section{PACIFIC JOURNAL OF MATHEMATICS}

\section{EDITORS}

DONALD BABBITT (Managing Editor)

University of Galifornia

Los Angeles, California 90024

HUGO RossI

University of Utah

Salt Lake City, UT 84112

C. C. MOORE AND ANDREW OGG

University of California

Berkeley, CA 94720
J. DUGUNDJI

Department of Mathematics University of Southern California Los Angeles, California 90007

R. FINN AND J. MILGRAM Stanford University

Stanford, California 94305

ASSOCIATE EDITORS

E. F. BECKENBACH

B. H. NeumanN

F. WOLF

K. YosHidA

\section{SUPPORTING INSTITUTIONS}

UNIVERSITY OF BRITISH COLUMBIA UNIVERSITY OF SOUTHERN CALIFONIA CALIFORNIA INSTITUTE OF TECHNOLOGY UNIVERSITY OF CALIFORNIA MONTANA STATE UNIVERSITY STANFORD UNIVERSITY UNIVERSITY OF HAWAII UNIVERSITY OF NEVADA, RENO UNIVERSITY OF TOKYO U'NIVERSITY OF UTAH NEW MEXICO STATE UNIVERSITY WASHINGTON STATE UNIVERSITY OREGON STATE UNIVERSITY UNIVERSITY OF OREGON UNIVERSITY OF WASHINGTON 


\section{Pacific Journal of Mathematics}

\section{Vol. 90, No. $1 \quad$ September, 1980}

Shashi Prabha Arya and M. K. Singal, On the locally countable sum

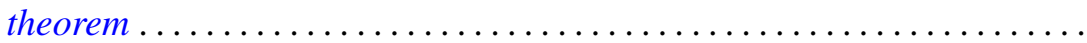

John Theodore Baldwin and David William Kueker, Ramsey quantifiers and

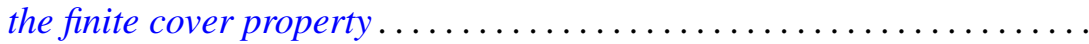

Richard Body and Roy Rene Douglas, Unique factorization of rational

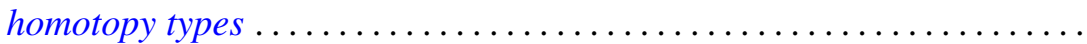

Ethan Bolker and Ben G. Roth, When is a bipartite graph a rigid

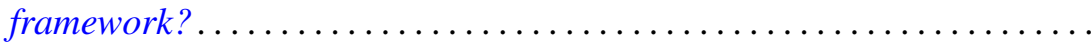

Alicia B. Winslow, Continua in the Stone-Čech remainder of $R^{2} \ldots \ldots \ldots$

Richard D. Carmichael and Elmer Kinji Hayashi, Analytic functions in tubes which are representable by Fourier-Laplace integrals ..............

Stephen D. Cohen, The Galois group of a polynomial with two indeterminate coefficients ..............................

Russell Allan Johnson, Strong liftings commuting with minimal distal

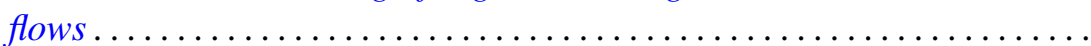

Elgin Harold Johnston, The boundary modulus of continuity of harmonic

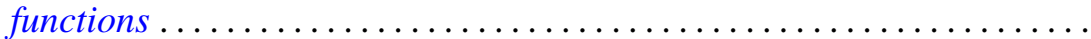

Akio Kawauchi and Takao Matumoto, An estimate of infinite cyclic

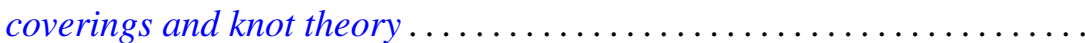

Keith Milo Kendig, Moiré phenomena in algebraic geometry: rational alternations in $\mathbf{R}^{2}$...

Roger T. Lewis and Lynne C. Wright, Comparison and oscillation criteria for selfadjoint vector-matrix differential equations .

Teck Cheong Lim, Asymptotic centers and nonexpansive mappings in conjugate Banach spaces .......................

David John Lutzer and Robert Allen McCoy, Category in function spaces.

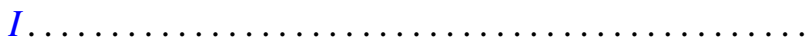

Richard A. Mollin, Induced p-elements in the Schur group ...

Jonathan Simon, Wirtinger approximations and the knot groups of $F^{n}$ in $S^{n+2}$

Robert L. Snider, The zero divisor conjecture for some solvable groups...

H. M. (Hari Mohan) Srivastava, A note on the Konhauser sets of biorthogonal polynomials suggested by the Laguerre polynomials...

Nicholas Th. Varopoulos, A probabilistic proof of the Garnett-Jones theorem on BMO.

Frank Arvey Wattenberg, $[0, \infty]$-valued, translation invariant measures on $N$ and the Dedekind completion of ${ }^{*} R \ldots \ldots \ldots \ldots . .$. 